\title{
Close binaries and common envelopes
}

\author{
David Jones, Jorge García-Rojas, Ondřej \\ Pejcha and Roger Wesson report on \\ their RAS Specialist Discussion Meeting \\ exploring "Common envelope evolution and \\ post-common-envelope systems".
}

\footnotetext{
A slow- and intermediate-mass stars evolve they expand to b ecome red giants, reaching maximum radii of hundreds of times that of the Sun, before shedding their outer layers leaving behind a white dwarf (WD). It has always been challenging to understand how such WDs can exist in close-binary systems, where the orbital separation between the two components is much smaller than the radius that the progenitor star of the WD would have reached while a red giant. It was not until Paczynski (1976), that a satisfactory evolutionary scenario was put forward: the common envelope (CE) phase (although he credited Ostriker and Webbink with the idea). In a common envelope evolution, as the WD progenitor grows to become a red giant it begins to transfer material on to its companion in a runaway process that leads to its engulfment. The core of the red giant and its companion are then orbiting inside a non-corotating "common envelope" of material formed from the outer layers of the expanding red giant. Drag forces ultimately lead to the ejection of the envelope material and the in-spiral of the stars to much tighter orbits, perhaps even to a merger (figure 1).

The CE (or some variant thereof, e.g. Soker 2015) has since become the accepted scenario for the formation of all compact binaries with a WD component. However, despite more than 40 years of vigorous observational and theoretical study, the CE still remains one of the most poorly understood phases of stellar evolution (Ivanova et al. 2013a). The RAS meeting "Common envelope evolution and post-common-envelope systems", held on 11 October 2019, aimed to bring together theorists and observers working on various aspects of CE and post-CE evolution, in order to drive forward our understanding of this fascinating and important phase of binary evolution.

The meeting itself began with a wonderful review by Robert Izzard (University of Surrey, UK), introducing the CE phase and its impact on nucleosynthesis. Sun-like stars are the main agents responsible for enriching the interstellar medium in chemical elements that include lithium, carbon, nitrogen and a whole host of heavier s-process elements. However, mass transfer in a binary system - and particularly the strong mass transfer and loss experienced during the CE phase - can have dramatic effects on the yields of these elements from a given star, essentially cutting short its nucleosynthetic evolution. This means that chemical yields from population synthesis models must take into account the effect of binarity above and beyond simply considering which systems might lead to a supernova explosion, and the related shockwave and r-process nucleosynthesis (Izzard \& Tout 2003, Tout 2006).
}

1 A cartoon representation of the common envelope, beginning with the Rochelobe overflow of the more massive component (on the left) in the upper panel, which leads to the engulfment of the companion in the middle panel. Drag forces, as the companion and primary's core orbit inside the common envelope, lead to the transfer of energy and angular momentum into the envelope causing the binary orbit to shrink. If enough orbital energy is transferred to the envelope it will be ejected (lower left panel), likely leading to the formation of a planetary nebula, otherwise the two stars will continue to spiral-in until merger (lower right panel). (P Hall, Hall etal. 2013)

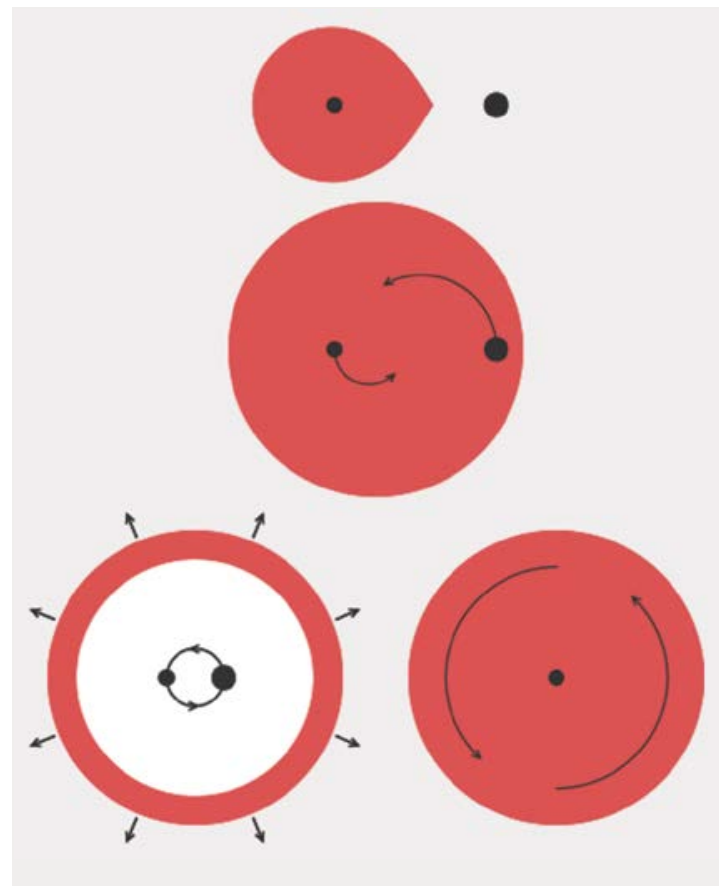

Planetary nebulae

The meeting continued with a series of talks on the young est (surviving) post-CE binary systems: those at the hearts of planetary nebulae (PNe). In classical single-star evolution, PNe are the glowing shells of gas and dust comprising material lost, in the form of a slow, dense stellar wind, by the central star while it was a red giant. This material is then ionized by the now exposed and rapidly heating core (which is en route to becoming a white dwarf). Post-CE PNe, on the other hand, are formed not from the remnants of a stellar wind but from the ejected envelope itself. Given the relatively short visibility lifetime of the nebulae ( $\tau \sim 30000 y r s$ ), this guarantees that the central binary has only recently left the CE and, as such, is "fresh-out-of-theoven" and has not yet had time to adjust (Jones \& Boffin 2017). Indeed, the discovery of a close-binary star at the heart of a PN was considered by Paczynski as key evidence for the existence of a CE phase, with the first being found the same year as his seminal paper (Bond 1976).

Henri M J Boffin (European Southern Observatory, Germany) highlighted that PNe offer a unique opportunity, not only to study the immediate products of the CE but also the pre-CE evolution. Many post-CE PNe are found to display polar outflows which are thought to trace a phase of stable mass transfer prior to the dynamical event that constitutes the CE (Boffin \& Jones 2019). This hypothesis is further supported by the discovery of carbon contamination in the companion to the central star of the Necklace Nebula (Miszalski et al. 2013), most likely from carbon-enhanced material transferred from the central star while it was an asymptotic giant branch star. Furthermore, previous studies of the main-sequence companions of post-CE PN central stars have found that all are "inflated" with respect to single stars of the same spectral type (Jones et al. 2015). Some of this inflation could be the result of the intense levels of irradiation experienced by these stars, but it is far more likely to be the result of rapid mass transfer that knocked the star out of thermal equilibrium (Prialnik \& Livio 1985).

Given that this accretion is unlikely to have occurred during the CE spiral-in, when strong entropy gradients form between the star and the surrounding envelope, it is thought to be the result of mass transfer prior to the CE. This hypothesis is supported by the observed ages of jets in some post-CE PNe, which are found to be older than the central nebular regions, for example in the archetype of the class, Fleming 1 (Boffin et al. 2012, and figure 2). 


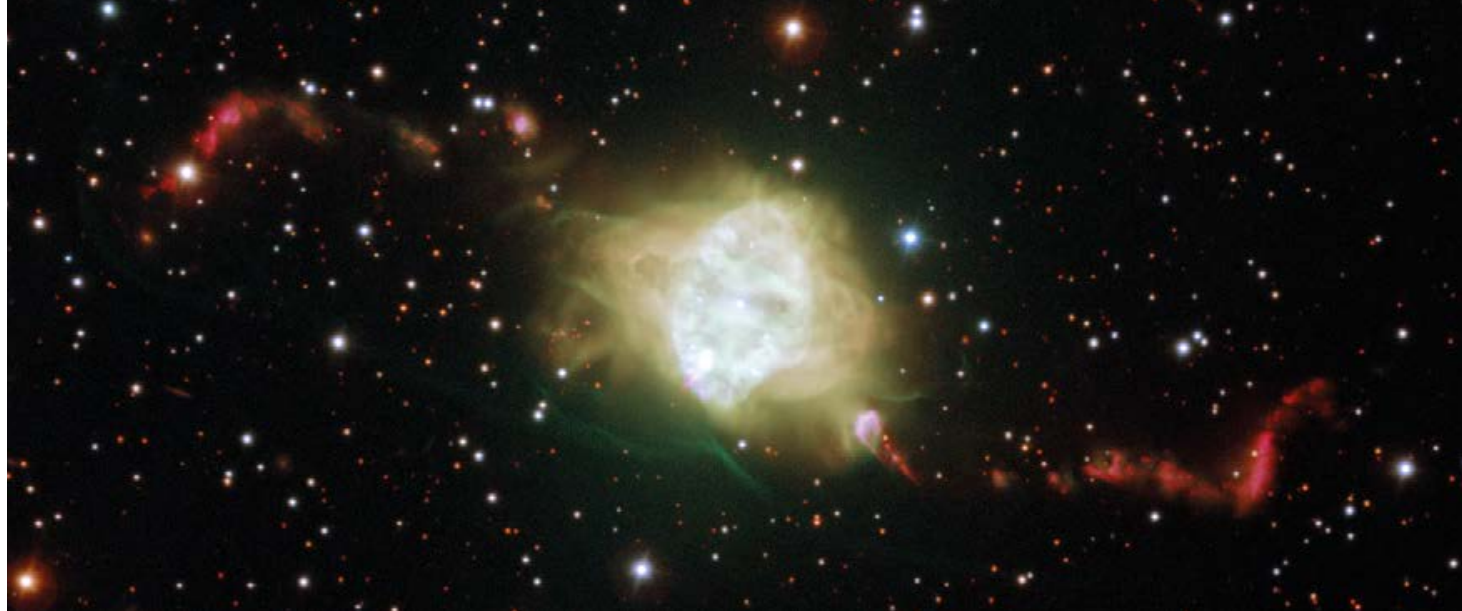

Miguel Santander-García (Observatorio Astronómico Nacional, Spain) continued the PN-theme by discussing his survey looking for molecular material in post-CE PNe. Recent studies had shown that post-CE PNe showed anomalously low ionized masses compared to the general population of $\mathrm{PNe}$, with the masses of the handful of post-CE PNe that have been subjected to sufficiently detailed study presenting with ionized masses more akin to novae (Corradi et al. 2015). Our current understanding of the CE would seem to indicate that post-CE PNe should be at least as massive as PNe originating from single stars, if not more so. It is important to test whether this "missing mass" problem is real and, as such, an important window into understanding the $\mathrm{CE}$, or if the material is simply hidden in another state, for example, in the molecules Santander-García is searching for (Santander-García et al. 2019). The preliminary results of the survey seem to indicate that, on a statistical level, the mass discrepancy between post-CE PNe and the general PN population is far less dramatic than previously indicated. However, there does seem to be some correlation between the binary components and the nebular mass, where systems with main-sequence companions appear to show significantly lower nebular masses than those with a degenerate companion. This may offer a route towards understanding the evolution towards close double-degenerate systems which are thought to avoid a CE when the more massive component evolves, instead experiencing a phase of stable mass transfer, only passing through the CE when the second component evolves from the main sequence (Woods et al. 2012).

Understanding the formation of close doubledegenerate systems represents a key challenge in modern astrophysics because these systems are important sources of gravitational waves for future space-based missions such as LISA (Korol et al. 2018). Furthermore, the mergers of double WD systems may represent a pathway towards Type Ia supernovae (Santander-García et al. 2015), which have been critical tools used to probe the expansion rate of the universe at high redshift (Riess et al. 1998, Perlmutter et al. 1999). This work was ultimately awarded the 2011 Nobel Prize for Physics, in spite of the fact that the progenitors of Type Ia supernovae are still unclear, as is the physics of what makes them such excellent standard candles (Phillips 1993).

\section{Extreme companion masses}

As the morning session continued, the talks moved towards more evolved post-CE binaries, beginning with the search for WDs with early-type main-sequence companions led by Steven Parsons (University of Sheffield, UK). There are now thousands of post-CE systems known comprising a WD and main-sequence star, but only a handful where the companion main-sequence star is more massive than half a solar mass. This dearth of massive companions is likely to arise from the intrinsic difficulty of identifying such systems: such bright companions outshine the WD components of the binary by several orders of magnitude in the optical range (Parsons et al. 2016). Radial velocity surveys, however, may hold the key to revealing the otherwise hidden WDs, with subsequent space-based ultraviolet observations providing critical confirmation. Importantly, the new systems with massive main-sequence companions discovered by Parsons can be used to probe how the CE differs in the low and high mass regimes (Brown et al. 2019), particularly given that previous studies have indicated that there may be a correlation between the efficiency of the CE (i.e. what fraction of the binary's orbital energy is used to eject the envelope) and the pre-CE parameters (for example, the masses; Wilson \& Nordhaus 2019). Additionally, post-CE systems with high-mass companions can place critical constraints on the formation scenarios for Type Ia supernovae (Parsons et al. 2016), as these binaries represent the strongest single-degenerate progenitor candidates. Given that the companions are massive, one would expect the WD progenitor to have also been rather massive and thus lead to a massive WD remnant which could feasibly grow, via a future mass transfer episode, reaching the Chandrasekhar mass and possibly explode as a Type Ia supernova.

At the other end of the companion mass range, Sarah Casewell (University of Leicester, UK) highlighted that only eight post-CE systems comprising a detached WD and brown dwarf (an object more massive than a gas giant planet but still below the nuclear burning limit; e.g. Maxted et al. 2006) are known while a similar number are found in cataclysmic variables or in hot subdwarf-brown dwarf pairs. Such systems place important constraints on the minimum mass to be able to survive a CE event (Casewell et al. 2018, Parsons et al. 2017), as well as a unique opportunity to study the atmospheres of brown dwarfs given that the high levels of irradiation from their companion WDs lead to the production of metal emission lines (Casewell et al. 2015, Longstaff et al. 2017).

\section{Mergers}

Thus far, we have only considered CEs where the binary system manages to impart sufficient energy into the envelope for it to be ejected before the two stars merge. However, it is clear that this will not always be the case and an appreciable number of systems will merge during the CE (Kochanek et al. 2014). It is only relatively recently that the transient events associated with such "failed" CEs were identified - still using their original mis-assigned moniker: luminous red novae (LRNe; Ivanova et al. 2013b). It is interesting that the names of perhaps the two most direct products of the CE - PNe and LRNe - are both misnomers; just as PNe bear no relation to planets, LRNe are completely unrelated to novae. The name is simply a reflection of the fact that they were first discovered as red transients with peak luminosities between those of novae and supernovae.

The first three talks of the afternoon were dedicated to observations and simulations of $\mathrm{LRNe}$, beginning with a review by Nadeja Blagorodnova (Radboud University,
2 The post-commonenvelope planetary nebula Fleming 1. The observed jets have been shown to predate the central nebula by a few thousand years, while the central star is a double-degenerate binary with an orbital period of 1.195 days (Boffin et al. 2012). (ESO/H Boffin) 
